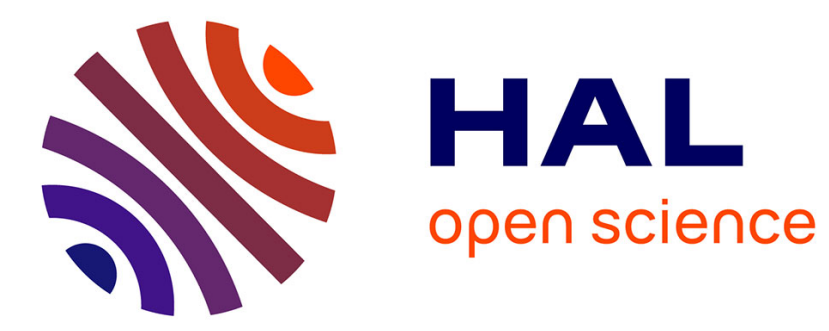

\title{
PCQM: A Full-Reference Quality Metric for Colored 3D Point Clouds
}

\author{
Gabriel Meynet, Yana Nehmé, Julie Digne, Guillaume Lavoué
}

\section{To cite this version:}

Gabriel Meynet, Yana Nehmé, Julie Digne, Guillaume Lavoué. PCQM: A Full-Reference Quality Metric for Colored 3D Point Clouds. 12th International Conference on Quality of Multimedia Experience (QoMEX 2020), May 2020, Athlone, Ireland. 10.1109/QoMEX48832.2020.9123147 . hal-02529668

\section{HAL Id: hal-02529668 https://hal.science/hal-02529668}

Submitted on 2 Apr 2020

HAL is a multi-disciplinary open access archive for the deposit and dissemination of scientific research documents, whether they are published or not. The documents may come from teaching and research institutions in France or abroad, or from public or private research centers.
L'archive ouverte pluridisciplinaire HAL, est destinée au dépôt et à la diffusion de documents scientifiques de niveau recherche, publiés ou non, émanant des établissements d'enseignement et de recherche français ou étrangers, des laboratoires publics ou privés. 


\title{
PCQM: A Full-Reference Quality Metric for Colored 3D Point Clouds
}

\author{
Gabriel Meynet, Yana Nehmé, Julie Digne and Guillaume Lavoué \\ CNRS, Univ. Lyon \\ LIRIS, UMR5205, F-69622 France \\ \{gabriel.meynet, yana.nehme, julie.digne, guillaume.lavoue\}@liris.cnrs.fr
}

\begin{abstract}
D point clouds constitute an emerging multimedia content, now used in a wide range of applications. The main drawback of this representation is the size of the data since typical point clouds may contain millions of points, usually associated with both geometry and color information. Consequently, a significant amount of work has been devoted to the efficient compression of this representation. Lossy compression leads to a degradation of the data and thus impacts the visual quality of the displayed content. In that context, predicting perceived visual quality computationally is essential for the optimization and evaluation of compression algorithms. In this paper, we introduce PCQM, a full-reference objective metric for visual quality assessment of 3D point clouds. The metric is an optimallyweighted linear combination of geometry-based and color-based features. We evaluate its performance on an open subjective dataset of colored point clouds compressed by several algorithms; the proposed quality assessment approach outperforms all previous metrics in terms of correlation with mean opinion scores.
\end{abstract}

Index Terms-Visual Quality Assessment, Point Cloud, Objective Metric.

\section{INTRODUCTION}

With the increasing capability of 3D data acquisition devices (RGBD cameras, 3D scanners, photometric), point clouds are becoming a popular representation in a wide range of applications, from manufacturing and construction to 3D telepresence. In this representation, a 3D asset simply consists in a collection of points; each point has geometric coordinates $(x, y, z)$ but may also contain a number of other attributes such as color, reflectance and surface normals. In many applications, point clouds are preferred to polygonal meshes for representing 3D assets, thanks to their flexibility and simplicity. However, the main disadvantage of point clouds is the size of the data which may be huge. Indeed, since there is not explicit surface information that could allow texture mapping, then a high precision in geometric details or colors require an increased number of points. As an example, in telepresence systems, typical sizes of point clouds are between 100,000 and $10,000,000$ points [1]. As a consequence of this large amount of data, a significant amount of work has been devoted to the efficient compression of this representation [1]-[5]. These algorithms usually compress both the geometry and color information. Since they are lossy, they introduce geometry and color distortions that impact the perceived quality of the decoded data. Accurate objective quality metrics are thus needed to evaluate this visual quality and drive compression algorithms.

In this context, we propose a full-reference objective quality metric for 3D point clouds with color attributes. We select several geometry-based and color-based features. An optimal subset of these features is then selected and combined by logistic regression. Geometry-based features are based on curvature analysis and are borrowed from the recent PC-MSDM metric [6]. For the color-based features, we extended for point clouds the lightness, chroma, and hue comparisons proposed in [7]. To our knowledge, our proposed metric is the first attempt to integrate both geometry and color information for quality assessment of point clouds. We conduct experiments on a recent benchmark proposed by Javaheri et al. [8], composed of 54 colored points clouds, created from 6 references compressed with three different compression algorithms. We evaluate the performance of each feature individually, and of the proposed linear model and compare them to state-of-the-art approaches. Our contributions are the following:

1) We extend the set of image color features proposed in [7] to 3D point clouds.

2) We evaluate individually the performance of a set of perceptually-relevant curvature-based and color-based features for predicting the perceived visual quality of 3D point clouds.

3) We provide an optimized linear model, which outperforms state-of-the-art metrics.

4) The source code is made publicly available ${ }^{1}$ to support further research in this area.

The following section details the existing work on objective quality assessment of 2D images and 3D point clouds. Section III details the proposed metric, while section IV presents the results.

\section{RELATED WORK}

Image quality metrics. In the field of 2D image processing, the research on objective quality assessment metrics is highly developed [9]. Bottom-up techniques try to mimic the lowlevel mechanisms of the human visual system (HVS) such as the contrast sensitivity function (CSF), usually modelled by a band-pass filter, and the visual masking effects that define the fact that one visual pattern can hide the visibility of another. They include the Sarnoff Visual Discrimination

\footnotetext{
${ }^{1}$ https://github.com/MEPP-team/PCQM
} 
Model (VDM) [10], the Visible Difference Predictor (VDP) [11] and their extensions (e.g., [12]). Top-down approaches do not rely upon the visual system principles but instead propose some signal fidelity criteria expected to correlate well with the perceptual quality such as the Structural SIMilarity index (SSIM) [13]. A large number of top-down image quality metrics have been proposed since then [14], [15]. Note that they are mostly considering the sole luminance information; only a few works (e.g., [7], [16]) are integrating chromatic information. Note that, the most recent top-down metrics are data-driven, i.e., they are learned on subjective data obtained from user studies [17]-[21].

Point cloud quality metrics. The field of point cloud quality assessment is still an emerging field. Simplest metrics include point-to-point and point-to-plane distances [22]. For each point of the content under evaluation, its closest point from the reference content is computed using nearest neighbour search. The point-to-point distance refers to the distance between those two points, while the point-to-plane distance refers to the projection of the distance vector along an average normal vector. These simple distances show good correlation results with subjective opinions for simple test content (e.g. one single type of degradation, such as in [23]). However, they report bad results for most of subjective datasets [24]-[26]. Very recently, Alexiou et al. [25] proposed a metric based on differences of normal orientations and Meynet et al. [6] proposed a metric integrating the curvature information. Both these metrics demonstrated improved performance. Several authors also computed 2D image metrics on a set of snapshots around the point clouds and reported correct correlations with subjective scores [26]-[28]. However, this kind of projectionbased approaches have the drawback of being hardly dependent of the rendering used to create the 2D views. Surprisingly, whereas several subjective studies involve colored point clouds [8], [23], [26], [28], [29], no attempt has yet be made to create a quality metric handling both geometry and color attributes. Note that several quality metrics have also been designed to address 3D content represented by triangulated surfaces. They attempt to predict the visual fidelity of a distorted 3D mesh with respect to a reference one [30]-[33]. They are mostly top-down approaches that rely on different geometric attributes (e.g., dihedral angles, curvatures). A few attempts have been made to integrate distortions on both geometry and texture maps [34].

Overall, except projection-based approaches, there is no existing point cloud quality metric that take into account both geometry and color.

\section{Proposed Visual Quality Metric}

\section{A. Overview}

Assessing computationally the visual quality of a 3D content composed of both geometry and color information is a particularly difficult task. Indeed, once some geometry and color features have been computed, the central question is how to combine them? This problem is also complicated by the fact that the optimal combination strongly depends on the rendering used when displaying the content. For instance, for the particular case of 3D meshes with diffuse textures, Guo et al. [34] showed that the absence of shading during the rendering (i.e., the pixel colors depend only on the texture colors, without any lighting effect) tends to mask the geometry artifacts and emphasize the color artifacts, as opposed to a rendering with shading. To resolve this complex combination problem, we consider a data-driven approach: we first select and compute different features $f_{i}$, a part of them being related to geometry and the other to color. Our Point Cloud Quality Metric (PCQM) is then computed as a linear combination (computed through logistic regression) of an optimal subset of these features.

Before computing these features (see Section III-D), we first establish a correspondence between the distorted point cloud $D$ and the reference point cloud $R$ (see Section III-B) and we define a proper neighborhood for each point (see Section III-C).

\section{B. Correspondence Between Point Clouds}

The first step of our approach consists in establishing a correspondence between the point clouds being compared: for each point $p$ from $R$ we compute its corresponding point $\hat{p}$ on $D$. It amounts to projecting the points from $R$ onto $D$. We restrict the projection from $R$ onto $D$ since the sampling density of $R$ is the most relevant to capture the distortion information. Projecting points from $D$ to $R$ may lead to information loss (e.g., if $D$ is heavily subsampled).

To establish a correspondence, for each point $p \in R$, most of existing approaches [22], [25] simply consider the closest point $p^{D} \in D$ (i.e., the nearest neighbor of $p$ belonging to $D$ ), however this makes the correspondence strongly dependent on the point sampling density and may lead to inaccurate results. Instead, as in [6], we search, for each $p$, the projection $\hat{p}$ on the $3 \mathrm{D}$ surface subtended by $D$. To do so we consider a local least squares fitting of a quadric surface (illustrated in Figure 1). This quadric surface fitting is computed on the set of nearest neighbors $p_{i}^{D} \in D$ of $p$. First we estimate an approximate tangent plane using Principal Component Analysis, which gives us an orthonormal frame $\left(u_{x}, u_{y}, u_{z}\right)$ such that $u_{z}$ is aligned with an approximate normal to the surface. We take $p$ as the origin of the coordinate system. In this local frame, the neighbor $p_{i}^{D}$ of $p$ has coordinates $\left(x_{i}, y_{i}, z_{i}\right)$. We thus look for the quadric surface $Q(x, y)=a x^{2}+b y^{2}+c x y+d x+e y+f$ minimizing:

$$
\sum_{i}\left\|z_{i}-Q\left(x_{i}, y_{i}\right)\right\|^{2}
$$

Once the quadric surface $Q$ is fitted, $\hat{p}$ is given as $(0,0, Q(0,0))$ in the $\left(u_{x}, u_{y}, u_{z}\right)$ frame mentioned above. Note that for computing the nearest neighbors $p_{i}^{D}$ of $p$, we consider a spherical neighborhood as detailed in Section III-C.

This geometric correspondence between point clouds $R$ and $D$ allows us to compute geometry-based features (see 
Section III-D). However we also need to compute colorbased features and thus we need to establish also a color correspondence between point clouds; in other words, we need to assign a color for each $\hat{p}$. For this purpose, once the projected point $\hat{p}$ is determined, we simply assign it the color of its nearest neighbor on $D$. We implemented more sophisticated barycentric interpolation methods both in RGB and LAB color-spaces, however results were less accurate.

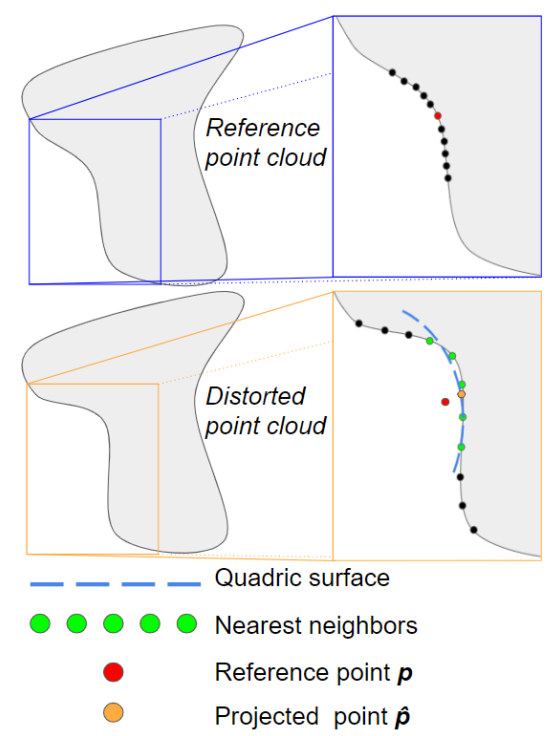

Fig. 1. Illustration of the point-to-surface correspondence computation.

\section{Neighborhood Computation}

The perceptually relevant features presented in Section III-D, as well as the quadric surface fitting presented above, are computed on local neighborhoods around each point $p$ of $R$. For a given scale $h$, we define the neighborhood $N(p, h)$ as the set of points belonging to the sphere with center $p$ and radius $h$. Features are then computed by considering curvature or color statistics over $N(p, h) \in R$ and $N(\hat{p}, h) \in D$. Note that we use $h$ for the computation of the features and $\frac{h}{2}$ for the quadric fitting.

\section{Perceptually Relevant Features}

1) Geometry-based features: For geometry-based features we consider those used in the $P C-M S D M$ metric [6] and derived from [32]. These features are based on the mean curvature information $\rho$, and have demonstrated good performance in predicting perceived visual quality. The curvature $\rho$ can be directly computed from the coefficients of the fitted quadric surfaces presented in Section III-B:

$$
\rho=\frac{\left(1+d^{2}\right) a+\left(1+e^{2}\right) b-4 a b c}{\left(1+e^{2}+d^{2}\right)^{\frac{3}{2}}}
$$

For each point $p$ from $R$, two curvature values are computed: $\rho_{p}$ computed using a quadric fitted over the local neighborhood $N\left(p, \frac{h}{2}\right) \in R$ and $\rho_{\hat{p}}$ computed using a quadric fitted over the local neighborhood $N\left(\hat{p}, \frac{h}{2}\right) \in D$. We then consider the three following features:

$$
\begin{aligned}
\text { Curvature comparison } & f_{1}^{p}=\frac{\left\|\mu_{p}^{\rho}-\mu_{\hat{p}}^{\rho}\right\|}{\max \left(\mu_{p}^{\rho}, \mu_{\hat{p}}^{\rho}\right)+k_{1}} \\
\text { Curvature contrast } \quad f_{2}^{p} & =\frac{\left\|\sigma_{p}^{\rho}-\sigma_{\hat{p}}^{\rho}\right\|}{\max \left(\sigma_{p}^{\rho}, \sigma_{\hat{p}}^{\rho}\right)+k_{2}} \\
\text { Curvature structure } \quad f_{3}^{p} & =\frac{\left\|\sigma_{p}^{\rho} \sigma_{\hat{p}}^{\rho}-\sigma_{p \hat{p}}^{\rho}\right\|}{\sigma_{p}^{\rho} \sigma_{\hat{p}}^{\rho}+k_{3}}
\end{aligned}
$$

where $k_{i}$ are constants to avoid instability when denominators are close to zero (they have been fixed to 1.0 as in [6]). $\mu_{p}^{\rho}, \mu_{\hat{p}}^{\rho}$ are Gaussian-weighted averages of curvature over the 3D points belonging to neighborhoods $N(p, h) \in R$ and $N(\hat{p}, h) \in D$ respectively. Similarly $\sigma_{p}^{\rho}, \sigma_{\hat{p}}^{\rho}$ and $\sigma_{p \hat{p}}^{\rho}$ are standard deviations and covariance of curvature over these neighborhoods.

2) Color-based features: Since no color feature have yet been introduced by the scientific community for quality evaluation of point clouds, we propose to extend the recent work from Lissner et al. [7], who proposed several features for the quality evaluation of $2 \mathrm{D}$ images. Their features have the benefit of taking into account not only the luminance but also the chromatic components.

As the authors, we first convert the RGB values to the perceptual color space LAB2000HL, introduced in [35]. Each point $p$ is thus associated to a lightness $L_{p}$ and two chromatic values $a_{p}$ and $b_{p}$. Similarly to [7], we define the chroma $c_{p}$ as $c_{p}=\sqrt{a_{p}^{2}+b_{p}^{2}}$. Our features are then defined as follows:

$$
\begin{aligned}
\text { Lightness comparison } & f_{4}^{p}=\frac{1}{k_{4} \cdot\left(\mu_{p}^{L}-\mu_{\hat{p}}^{L}\right)^{2}+1} \\
\text { Lightness contrast } & f_{5}^{p}=\frac{2 \sigma_{p}^{L} \sigma_{\hat{p}}^{L}+k_{5}}{\sigma_{p}^{L^{2}}+\sigma_{\hat{p}}^{L^{2}}+k_{5}} \\
\text { Lightness structure } & f_{6}^{p}=\frac{\sigma_{p \hat{p}}^{L}+k_{6}}{\sigma_{p}^{L} \sigma_{\hat{p}}^{L}+k_{6}} \\
\text { Chroma comparison } & f_{7}^{p}=\frac{1}{k_{7} \cdot\left(\mu_{p}^{c}-\mu_{\hat{p}}^{c}\right)^{2}+1} \\
\text { Hue comparison } & f_{8}^{p}=\frac{1}{k_{8} \cdot \overline{\Delta H_{p \hat{p}}}+1}
\end{aligned}
$$

with $\Delta H_{p \hat{p}}=\sqrt{\left(a_{p}-a_{\hat{p}}\right)^{2}+\left(b_{p}-b_{\hat{p}}\right)^{2}-\left(c_{p}-c_{\hat{p}}\right)^{2}}$ and $\overline{\Delta H_{p \hat{p}}}$ is the Gaussian-weighed average over $N(p, h)$. Constants $k_{i}$ have been fixed as the same values as in [7].

Note that, for each of these color features, we apply the transform $f_{i}^{p}=1-f_{i}^{p}$ to obtain a distance instead of a similarity index (i.e., a value of 0 means that there is no local distortion). Those features are then coherent with the geometry-based features. 


\section{E. Combining Features Into a Perceptual Quality Score}

The height local features $f_{i}^{p}$ presented above are computed for each point $p$, and are in $[0,1]$. In order to obtain global features $f_{i}$ we aggregate local values by taking the means of the distributions (this corresponds to an average pooling):

$$
f_{i}=\frac{1}{|R|} \sum_{p \in R} f_{i}^{p}
$$

Our metric is then defined as combination of these features $f_{i}$, i.e., as a mapping function from the feature space $\mathbf{f}$ to a scalar $\in[0,1]$. It is hard to anticipate what combination model would provide the best and more stable results. E.g., Lissner et al. [7] combine their color features using a factorial model, while Meynet et al. [6] consider a linear model for curvature-based features. In this work, to make the optimization easier, we consider a linear model, optimized through logistic regression. Our full reference metric PCQM, is thus defined as follows:

$$
P C Q M=\sum_{i \in S} w_{i} f_{i}
$$

$S$ is the set of indices of features of our linear model. The optimal subset of features and corresponding weights are determined through cross-validation, based on results from a subjective study (see Section IV-C2).

\section{RESULTS AND EVALUATION}

\section{A. Database}

To train and evaluate our metric, we need a dataset of distorted stimuli with mean opinion scores (MOS) obtained through a subjective experiment. These exist few publiclyavailable subject-rated databases of colored $3 \mathrm{D}$ point clouds [8], [23], [28]. We considered the IST Rendering Point Cloud Dataset $[8]^{1}$. Six colored point clouds from MPEG repository (with number of points ranging from 272,684 to $4,848,745$ ) have been compressed with three different codecs, each at three different rates representing low, medium and high quality. Octree-pruning, MPEG G-PCC and MPEG V-PCC codecs have been used. In total, the dataset thus contains 54 point clouds, each rated by 20 subjects using a DSIS subjective methodology. Note that three rendering methods (and thus three rating sessions) were used: $3 D$ points without color, reconstructed $3 D$ meshes without color and $3 D$ points with colors. Since our metric focus on colored point clouds, we consider MOS values from the latter session.

\section{B. Parameter of the features}

The only parameter of our metric is the scale $h$ used to compute the neighborhoods. This parameter actually defines the scale of the distortions that we want to capture and is linked to the subjective test protocol. In our experiment we consider $h=0.008 \times B B$ with $B B$ the maximum bounding box length.

\footnotetext{
${ }^{1}$ https://github.com/AlirezaJav/IRPC-Dataset
}

\section{Results}

In what follows, the performance of the features and metrics is evaluated using the Spearman and Pearson correlation coefficients between the objective metric's values and the subjective scores. Correlations are computed after a logistic regression which provides a non-linear mapping between the objective and subjective scores.

1) Single feature prediction performance: We first evaluate the prediction performance of each feature separately. Figure 2 illustrates the correlations of each feature $f_{i}$ with MOS.

TABLE I

CORRELATIONS OF THE INDIVIDUAL FEATURES WITH SUBJECTIVE MEAN OPINION SCORES FROM [8].

\begin{tabular}{lccc}
\hline Feature & id & Pearson & Spearman \\
\hline Curvature comparison & $f_{1}$ & 0.75 & 0.61 \\
Curvature contrast & $f_{2}$ & 0.78 & 0.61 \\
Curvature structure & $f_{3}$ & 0.55 & 0.62 \\
Lightness comparison & $f_{4}$ & 0.76 & 0.81 \\
Lightness contrast & $f_{5}$ & 0.76 & 0.78 \\
Lightness structure & $f_{6}$ & 0.79 & 0.73 \\
Chroma comparison & $f_{7}$ & 0.64 & 0.74 \\
Hue comparison & $f_{8}$ & 0.61 & 0.59 \\
\hline
\end{tabular}

We can see from Figure 2 and Table I that most of features provide a correct correlation with the subjective scores. Best features are Lightness comparison $f_{4}$, contrast $f_{5}$ and structure $f_{6}$. Curvature comparison $f_{1}$ and contrast $f_{2}$ also provide a good Pearson correlation but low Spearman scores, indicating a good global tendency but more outliers in the predictions, as can be seen also in Figure 2. Chromatic features seem to be less relevant.

2) Linear model optimization: To optimize our linear model, we consider logistic regression. Since features may be redundant and in order to prevent over-fitting we do not consider all features but we select an optimal subset. For a given subset of features, the weights are optimized as follows: we split the dataset into two equal parts (each containing 27 distorted point clouds). One part is used to optimize the weights by logistic regression and the other is used for testing the obtained metric. The splitting is done according to the reference point clouds, insuring that the test set does not contain any of the point clouds used for training, regardless of the distortion. It amounts to selecting 3 reference models among 6 for training and using the rest for testing. We repeat this operation using the $\left(\begin{array}{l}6 \\ 3\end{array}\right)=20$ possible splittings, and report the average performance.

Since we have 8 features, there are $2^{8}-1=255$ possible combinations of features. We exhaustively search through all possible combinations, and select the one that generates the best average performance in term of Pearson and Spearman correlation. The best model that we finally obtain is composed of only three features: Curvature structure $\left(f_{3}\right)$, Lightness comparison $\left(f_{4}\right)$ and Lightness structure $\left(f_{6}\right)$. Results are reported in the section below. 

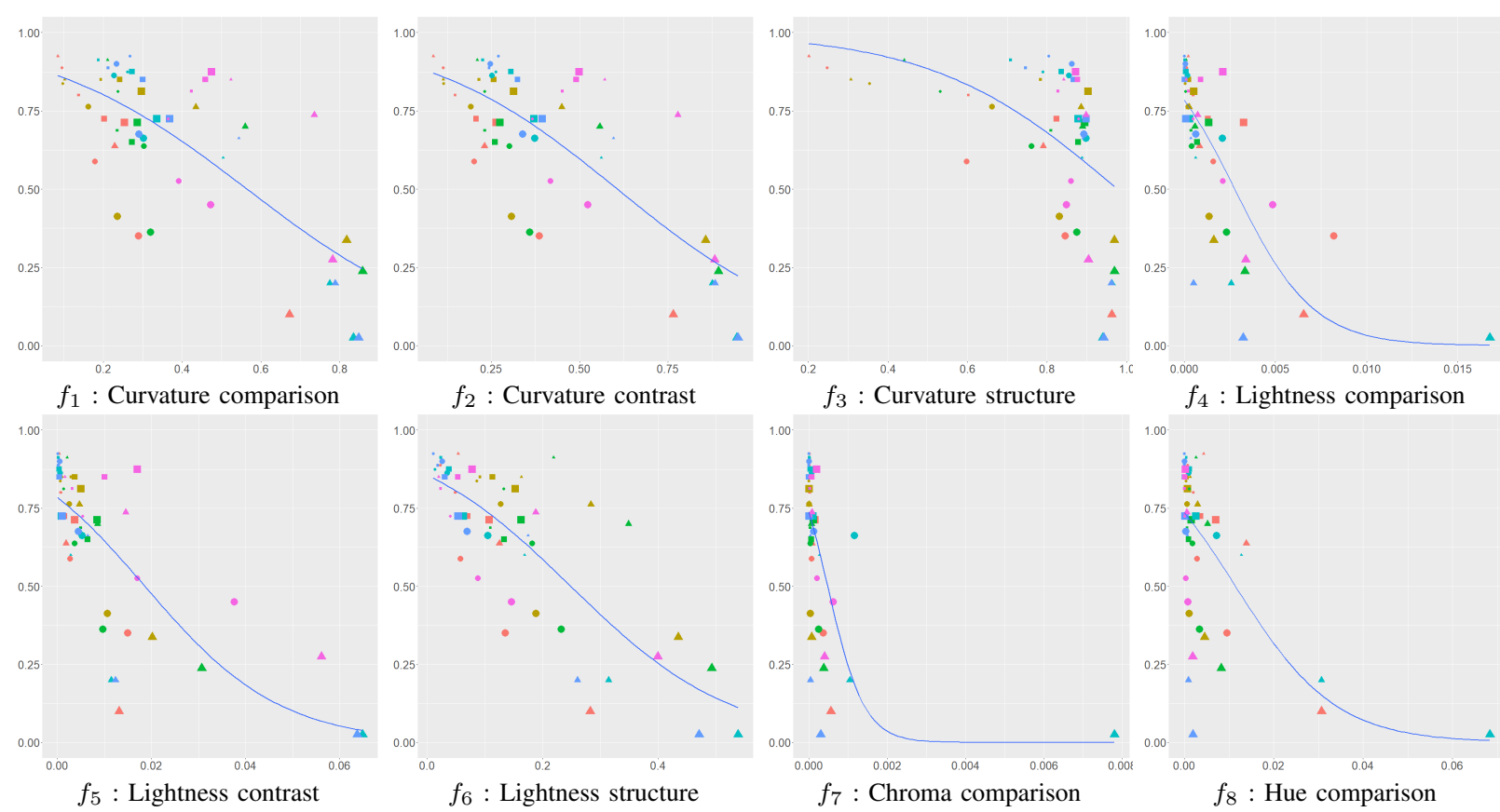

References

- Facade

- Frog

- House

- longdress

- loot

- Mask

$f_{4}$ : Lightness comparison

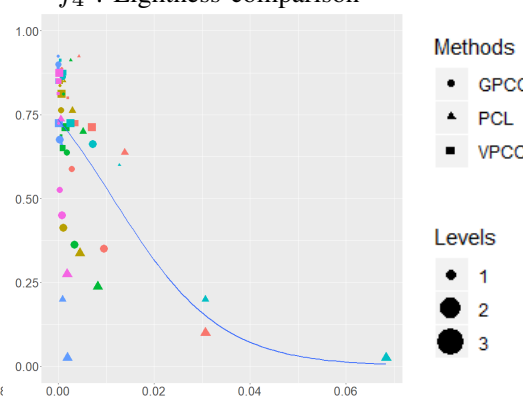

$f_{8}$ : Hue comparison

Fig. 2. Plots of the different features $f_{i}$ ( $X$ axis) versus the normalized mean opinion scores ( $Y$ axis). The fitted logistic function is displayed in blue.

3) Performance of our metric and comparisons: In Table II, we report the results of our metric $P C Q M$. We also include results of the best single feature $f_{5}$, and of two baselines : $d_{g e o}$ which is the average of the geometric distance (normalized by the bounding box length) between $p$ and $\hat{p}$ (computed by a simple nearest neighbor approach), and $d_{\text {color }}$ which is the average of their color distance (computed in LAB2000HL). For all these metrics, we report their average performance when computed on the 20 test sets, as described in the section above. We also report results of the state of the art metrics reported in [8] (we selected the best setting for each of them). Note that for these latter metrics (Point-to-Point, Point-toPlane and Plane-to-Plane), correlations were computed once over the whole dataset.

TABLE II

CORRELATIONS OF DIFFERENT METRICS WITH SUBJECTIVE MEAN OPINION SCORES FROM [8]. MEAN CORRELATION VALUES (AVERAGED OVER THE 20 TEST SETS) ARE REPORTED, EXCEPT FOR METRICS MARKED WITH A* FOR WHICH VALUES ARE REPRINTED FROM [8].

\begin{tabular}{lcc}
\hline Metric & Pearson & Spearman \\
\hline Best single feature $\left(f_{5}\right)$ & $0.814(\mathrm{SD}=0.098)$ & $0.787(\mathrm{SD}=0.095)$ \\
PCQM & $\mathbf{0 . 8 7 3}(\mathbf{S D}=\mathbf{0 . 0 4 7})$ & $\mathbf{0 . 8 0 7}(\mathbf{S D}=\mathbf{0 . 0 6 4})$ \\
$D_{\text {geo }}$ & $0.843(\mathrm{SD}=0.090)$ & $0.749(\mathrm{SD}=0.140)$ \\
$D_{\text {color }}$ & $0.724(\mathrm{SD}=0.091)$ & $0.665(\mathrm{SD}=0.098)$ \\
\hline Point-to-Point $(\mathrm{PSNR}) *$ & 0.788 & \\
Point-to-Plane $(\mathrm{PSNR}) *$ & 0.784 & \\
Plane-to-Plane (MAD)* & 0.244 & \\
\hline
\end{tabular}

Table II demonstrates that our metric performs very well. The best single feature $\left(f_{5}\right)$ provides also good results. Note that, in this setting (i.e., average correlations over 20 test sets of 27 point clouds), results for this feature are slightly different from those reported in Table I which are computed once over the whole dataset (of 54 point clouds).

Our geometric distance, $d_{g e o}$, provides also good average results on this dataset, but with high standard deviation. Its results seem to be better than the point-to-point distance reported in [8]. This difference may be due to the fact that we consider only an asymmetric distance (from $R$ to $D$ ). However, despite its good results on this dataset, the pure geometric distance has shown to behave very poorly in other datasets (e.g., [24]-[26]).

4) Recommended weights: We provide the recommended model for use by the scientific community. We averaged the weights of our linear models obtained for each of the 20 training subsets:

$$
P C Q M_{\text {rec }}=0.18 f_{3}+0.44 f_{4}+0.38 f_{6}
$$

Note that in order to reveal the relative importance of each feature, the weights presented in the equation above are scaled by the standard deviation of the features. Real unscaled weights are $0.0057,0.9771$, and 0.0172 , respectively for $f_{3}, f_{4}$ and $f_{6}$. Figure 3 illustrates subjective MOS against the metric values. The Pearson and Spearman correlations computed over the whole dataset are 0.90 and 0.83 , respectively. For the sake of comparison the figure also illustrates the baseline color distance $d_{\text {color }}($ Pearson=0.71 and Spearman=0.65).

\section{CONCLUSiON}

In this work, we presented a full reference metric for the quality assessment of colored 3D point clouds. Our metric is data-driven and is defined as a linear combination of an optimal subset of geometry-based and color-based features. Those features are computed thanks to accurate mechanisms 

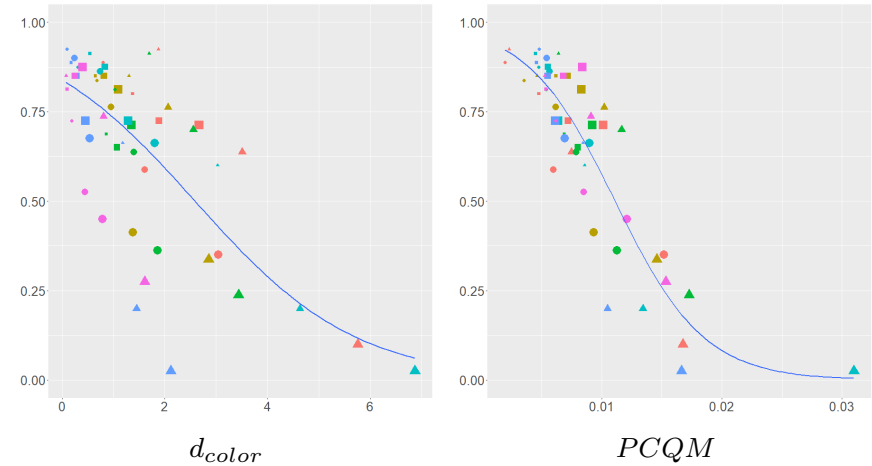

Fig. 3. Subjective MOS vs metric values for our metric $P C Q M$ and the baseline color distance $d_{\text {color }}$. The legend is the same as in Figure 2 .

for correspondence and neighborhood computation. Our experimental results show that color features, particularly Lightness information, plays an important role in the human perception of point clouds; they also demonstrate that geometry (represented by curvature) and color are complementary attributes since our optimal computational model includes both of them.

\section{ACKNOWLEDGMENT}

We are grateful to João Ascenso and Fernando Pereira for sharing their subjective dataset. This work was supported by French National Research Agency as part of ANR-PISCo project (ANR-17-CE33-0005).

\section{REFERENCES}

[1] S. Schwarz, M. Preda, V. Baroncini, M. Budagavi, P. Cesar, P. A. Chou, R. A. Cohen, M. Krivokuca, S. Lasserre, Z. Li, J. Llach, K. Mammou, R. Mekuria, O. Nakagami, E. Siahaan, A. Tabatabai, A. M. Tourapis, and V. Zakharchenko, "Emerging MPEG Standards for Point Cloud Compression," IEEE Journal on Emerging and Selected Topics in Circuits and Systems, vol. 9, no. 1, pp. 133-148, 2019.

[2] D. Thanou, P. A. Chou, and P. Frossard, "Graph-based compression of dynamic 3D point cloud sequences," IEEE Transactions on Image Processing, vol. 25, no. 4, pp. 1765-1778, 2016.

[3] Y. Huang, J. Peng, C. C. Kuo, and M. Gopi, "A generic scheme for progressive point cloud coding," IEEE Transactions on Visualization and Computer Graphics, vol. 14, no. 2, pp. 440-453, 2008.

[4] R. De Queiroz and P. A. Chou, "Transform Coding for Point Clouds Using a Gaussian Process Model," IEEE Transactions on Image Processing, vol. 7149, no. c, pp. 1-1, 2017.

[5] R. Mekuria, K. Blom, and P. Cesar, "Design, Implementation, and Evaluation of a Point Cloud Codec for Tele-Immersive Video," IEEE Transactions on Circuits and Systems for Video Technology, vol. 27, no. 4, pp. 828-842, 2017.

[6] G. Meynet, J. Digne, and G. Lavoué, "PC-MSDM : A quality metric for 3D point clouds," in International Conference on Quality of Multimedia Experience, 2019.

[7] I. Lissner, J. Preiss, P. Urban, M. S. Lichtenauer, and P. Zolliker, "Imagedifference prediction: From grayscale to color," IEEE Transactions on Image Processing, vol. 22, no. 2, pp. 435-446, 2013.

[8] A. Javaheri, C. Brites, F. Pereira, and J. Ascenso, "Point Cloud Rendering after Coding : Impacts on Subjective and Objective Quality," arXiv:1912.09137, pp. 1-13, 2019.

[9] Z. Wang and A. C. Bovik, Modern Image Quality Assessment. Morgan \& Claypool Publishers, jan 2006, vol. 2, no. 1.

[10] J. Lubin, "The use of psychophysical data and models in the analysis of display system performance," in Digital Images and Human Vision, A. B. Watson, Ed., oct 1993, pp. 163-178.

[11] S. Daly, "The visible differences predictor: an algorithm for the assessment of image fidelity," in Digital images and human vision, Andrew B. Watson, Ed. Cambridge: MIT Press, oct 1993, pp. 179-206.
[12] R. Mantiuk, K. J. Kim, A. G. Rempel, and W. Heidrich, "HDR-VDP-2," ACM Transactions on Graphics, vol. 30, no. 4, p. 1, jul 2011.

[13] Z. Wang, A. Bovik, H. Sheikh, and E. Simoncelli, "Image quality assessment: From error visibility to structural similarity," IEEE Transactions on Image Processing, vol. 13, no. 4, pp. 600-612, 2004.

[14] H. Sheikh and A. Bovik, "Image information and visual quality," IEEE Transactions on Image Processing, vol. 15, no. 2, pp. 430-444, feb 2006.

[15] L. Zhang, X. Mou, and D. Zhang, "FSIM: A Feature Similarity Index for Image Quality Assessment." IEEE Transactions on Image Processing, no. 99, pp. 1-1, jan 2011

[16] J. Preiss, F. Fernandes, and P. Urban, "Color-image quality assessment: From prediction to optimization," IEEE Transactions on Image Processing, vol. 23, no. 3, pp. 1366-1378, 2014.

[17] M. Narwaria and W. Lin, "SVD-Based Quality Metric for Image and Video Using Machine Learning," IEEE Transactions on Systems, Man, and Cybernetics, vol. 42, no. 2, pp. 347-364, 2012.

[18] Y. Liu, J. Wang, S. Cho, A. Finkelstein, and S. Rusinkiewicz, "A noreference metric for evaluating the quality of motion deblurring." $A C M$ Transactions on Graphics, vol. 32, no. 6, pp. 171-175, 2013.

[19] F. Gao, D. Tao, S. Member, and X. Gao, "Learning to Rank for Blind Image Quality Assessment," IEEE Trans. on Neural Networks and Learning Systems, pp. 1-30, 2015.

[20] H. Yeganeh and Z. Wang, "Objective quality assessment of tone-mapped images," IEEE Transactions on Image Processing, vol. 22, no. 2, pp. 657-667, 2013.

[21] S. Bosse, D. Maniry, K. R. Müller, T. Wiegand, and W. Samek, "Deep Neural Networks for No-Reference and Full-Reference Image Quality Assessment," IEEE Transactions on Image Processing, vol. 27, no. 1, pp. 206-219, 2018.

[22] D. Tian, H. Ochimizu, C. Feng, R. Cohen, and A. Vetro, "Geometric distortion metrics for point cloud compression," in International Conference on Image Processing, 2017, pp. 3460-3464.

[23] E. Zerman, P. Gao, C. Ozcinar, and A. Smolic, "Subjective and Objective Quality Assessment for Volumetric Video Compression," in $S \& T$ Electronic Imaging, Image Quality and System Performance XVI, 2019.

[24] T. Ebrahimi and E. Alexiou, "On the performance of metrics to predict quality in point cloud representations," Applications of Digital Image Processing XL, no. c, p. 53, 2017.

[25] E. Alexiou and T. Ebrahimi, "Point Cloud Quality Assessment Metric Based on Angular Similarity," 2018 IEEE International Conference on Multimedia and Expo (ICME), pp. 1-6, 2018.

[26] H. Su, Z. Duanmu, W. Liu, Q. Liu, and Z. Wang, "Perceptual Qualty Assessment of point Clouds," IEEE International Conference on Image Processing, pp. 3182-3186, 2019.

[27] E. Alexiou and T. Ebrahimi, "Exploiting user interactivity in quality assessment of point cloud imaging," International Conference on Quality of Multimedia Experience, pp. 3-8, 2019.

[28] Evangelos Alexiou, I. Viola, T. M. Borges, T. A. Fonseca, R. L. de Queiroz, and T. Ebrahimi, "A comprehensive study of the ratedistortion performance in MPEG point cloud compression," APSIPA Transactions on Signal and Information Processing, vol. 8, 2019.

[29] L. A. d. S. Cruz, E. Dumic, E. Alexiou, J. Prazeres, R. Duarte, M. Pereira, A. Pinheiro, and T. Ebrahimi, "Point cloud quality evaluation : Towards a definition for test conditions," International Conference on Quality of Multimedia Experience, 2019.

[30] M. Corsini, E. D. Gelasca, T. Ebrahimi, and M. Barni, "Watermarked 3D Mesh Quality Assessment," IEEE Transactions on Multimedia, vol. 9, no. 2, pp. 247-256, feb 2007.

[31] L. Váša and J. Rus, "Dihedral Angle Mesh Error: a fast perception correlated distortion measure for fixed connectivity triangle meshes," Computer Graphics Forum, vol. 31, no. 5, 2012.

[32] G. Lavoué, "A Multiscale Metric for 3D Mesh Visual Quality Assessment," Computer Graphics Forum, vol. 30, no. 5, pp. 1427-1437, 2011.

[33] Guillaume Lavoué, M. C. Larabi, and L. Váša, "On the Efficiency of Image Metrics for Evaluating the Visual Quality of 3D Models," IEEE Transactions on Visualization and Computer Graphics, 2016.

[34] J. Guo, V. Vidal, I. Cheng, A. Basu, A. Baskurt, and G. Lavoue, "Subjective and objective visual quality assessment of textured 3D meshes," ACM Transactions on Applied Perception, vol. 14, no. 2, 2016.

[35] I. Lissner and P. Urban, "Toward a Unified Color Space for PerceptionBased Image Processing," IEEE Transactions on Image Processing, vol. 21, no. 3, pp. 1153-1168, 2012. 